\title{
GLP-1 receptors exist in the parietal cortex, hypothalamus and medulla of human brains and the GLP-1 analogue liraglutide alters brain activity related to highly desirable food cues in individuals with diabetes: a crossover, randomised, placebo-controlled trial
}

\author{
Olivia M. Farr ${ }^{1} \cdot$ Michail Sofopoulos $^{2} \cdot$ Michael A. Tsoukas $^{1} \cdot$ Fadime Dincer $^{1} \cdot$ \\ Bindiya Thakkar ${ }^{1}$ - Ayse Sahin-Efe ${ }^{1}$ • Andreas Filippaios ${ }^{1}$ - Jennifer Bowers ${ }^{1}$. \\ Alexandra Srnka ${ }^{1}$ - Anna Gavrieli ${ }^{1}$ - Byung-Joon Ko ${ }^{1,3}$. Chrysoula Liakou ${ }^{2}$. \\ Nickole Kanyuch $^{1}$ - Sofia Tseleni-Balafouta ${ }^{4} \cdot$ Christos S. Mantzoros $^{1}$
}

Received: 5 November 2015 / Accepted: 4 January 2016 /Published online: 1 February 2016

(C) Springer-Verlag Berlin Heidelberg 2016

\begin{abstract}
Aims/hypothesis Liraglutide is a glucagon-like peptide-1 (GLP-1) analogue that has been demonstrated to successfully treat diabetes and promote weight loss. The mechanisms by which liraglutide confers weight loss remain to be fully clarified. Thus, we investigated whether GLP-1 receptors are expressed in human brains and whether liraglutide administration affects neural responses to food cues in diabetic individuals (primary outcome).

Methods In 22 consecutively studied human brains, expression of GLP-1 receptors in the hypothalamus, medulla oblongata and parietal cortex was examined using
\end{abstract}

Electronic supplementary material The online version of this article (doi:10.1007/s00125-016-3874-y) contains peer-reviewed but unedited supplementary material, which is available to authorised users.

Olivia M. Farr

ofarr@bidmc.harvard.edu

1 Division of Endocrinology, Beth Israel Deaconess Medical Center/ Harvard Medical School, 330 Brookline Ave, Stoneman 820, Boston, MA 02215, USA

2 Department of Pathology, St Savvas Anticancer-Oncology Hospital, Athens, Greece

3 Total Healthcare Center, Kangbuk Samsung Hospital, Sungkyunkwan University School of Medicine, Seoul, Republic of Korea

4 First Department of Pathology, University of Athens, Medical School, Athens, Greece immunohistochemistry. In a randomised (assigned by the pharmacy using a randomisation enrolment table), placebocontrolled, double-blind, crossover trial, 21 individuals with type 2 diabetes (18 included in analysis due to lack or poor quality of data) were treated with placebo and liraglutide for a total of 17 days each $(0.6 \mathrm{mg}$ for 7 days, $1.2 \mathrm{mg}$ for 7 days, and $1.8 \mathrm{mg}$ for 3 days). Participants were eligible if they had type 2 diabetes and were currently being treated with lifestyle changes or metformin. Participants, caregivers, people doing measurements and/or examinations, and people assessing the outcomes were blinded to the medication assignment. We studied metabolic changes as well as neurocognitive and neuroimaging (functional MRI) of responses to food cues at the clinical research centre of Beth Israel Deaconess Medical Center. Results Immunohistochemical analysis revealed the presence of GLP-1 receptors on neurons in the human hypothalamus, medulla and parietal cortex. Liraglutide decreased activation of the parietal cortex in response to highly desirable (vs less desirable) food images $(p<0.001$; effect size: placebo $0.53 \pm 0.24$, liraglutide $-0.47 \pm 0.18$ ). No significant adverse effects were noted. In a secondary analysis, we observed decreased activation in the insula and putamen, areas involved in the reward system. Furthermore, we showed that increased ratings of hunger and appetite correlated with increased brain activation in response to highly desirable food cues while on liraglutide, while ratings of nausea correlated with decreased brain activation.

Conclusions/interpretation For the first time, we demonstrate the presence of GLP-1 receptors in human brains. We also observe that liraglutide alters brain activity related to highly 
desirable food cues. Our data point to a central mechanism contributing to, or underlying, the effects of liraglutide on metabolism and weight loss. Future studies will be needed to confirm and extend these findings in larger samples of diabetic individuals and/or with the higher doses of liraglutide $(3 \mathrm{mg})$ recently approved for obesity.

Trial registration ClinicalTrials.gov NCT01562678

Funding The study was funded by Novo Nordisk, NIH UL1 RR025758 and 5T32HD052961.

Keywords Brain · Diabetes · fMRI - GLP-1 . Immunohistochemistry $\cdot$ Liraglutide $\cdot$ MRI

Abbreviations
$\begin{array}{ll}\text { BIDMC } & \text { Beth Israel Deaconess Medical Center } \\ \text { BOLD } & \text { Blood oxygenation level dependent } \\ \text { CRC } & \text { Clinical research centre } \\ \text { fMRI } & \text { Functional magnetic resonance imaging } \\ \text { GLP-1 } & \text { Glucagon-like peptide-1 } \\ \text { VAS } & \text { Visual analogue scales }\end{array}$

\section{Introduction}

Diabetes mellitus is a significant and growing problem worldwide affecting more than 25 million people in the USA, with an estimated lifetime prevalence of one-third for individuals born in 2000 [1]. A related condition, obesity, is also a growing problem in industrialised countries, affecting over onethird of the population, with an additional third being overweight [2]. There is a clear need for understanding the underlying mechanisms of and identifying effective therapies to treat type 2 diabetes and obesity.

Recently, incretin therapies have come to the forefront of potential treatments for type 2 diabetes and obesity [3]. The major source of endogenous glucagon-like peptide-1 (GLP-1) in humans is the intestinal L cell [3]. Accumulating evidence from both animal and human studies has shown that GLP-1 has an anorectic effect and may lead to weight reduction [4]. The GLP-1 analogue liraglutide has been approved by the US Food and Drug Administration, at a dose of $1.8 \mathrm{mg}$, for use in individuals with diabetes as an adjunct to diet and exercise [5]. Most recently, liraglutide $3.0 \mathrm{mg}$ has been approved for the treatment of obesity.

In addition to acting on pancreatic and gastric targets, GLP-1 and liraglutide may act on the brain to produce satiety and suppress food intake [6, 7]. In rats and monkeys, GLP-1 receptors have been identified in several areas of the brain, including the hypothalamic nuclei and brainstem [8]. In mice, the weight-reducing effects of liraglutide were confirmed to be induced centrally, as this effect was moderated by the presence of neuronal GLP-1 receptors [9]. GLP-1 receptors have been identified in the hypothalamus, striatum, midbrain and hindbrain of primates but expression of these receptors has not been studied in humans [10]. The central effects of liraglutide also remain to be confirmed in humans, in whom the control of feeding involves other brain areas such as the prefrontal cortex which is responsible for cognitive control and decision-making, a rather complicated system which cannot be studied in mice or rats. The reward system also differs between mice and humans. Thus, although GLP-1 has been found to act on the mesolimbic dopamine system of mice [11], this remains to be studied in humans.

In this study, we used immunohistochemistry to explore whether GLP-1 receptors exist in human brains. We also investigated whether liraglutide administration could change the functional activation (using functional MRI [fMRI]) of brain centres important in energy homeostasis. A low dose of liraglutide was administered in a 2.5 -week-long, dose-escalation, randomised, placebo-controlled, crossover study in diabetic individuals. To optimise this study, we gave the highest dosage $(1.8 \mathrm{mg}$ [as $3.0 \mathrm{mg}$ had not yet been approved]) for a short time period ( 3 days) so that although the study took place at steady state and after any nausea initially caused by the medication, no weight loss would have occurred at the end of the study period and changes in body weight, per se, would not have confounded the results.

\section{Methods}

Study 1 Samples of hypothalamus, medulla oblongata and parietal cortex were obtained from discarded tissue taken from 22 autopsies (13 men, 9 women, aged 34-95 years) carried out less than $24 \mathrm{~h}$ after death. The selected paraffin blocks were analysed for the presence of GLP-1 receptors using standard immunohistochemical methods, employing the antibody against the human GLP-1 receptor (MAb 3F52) as previously described [10-12]. See electronic supplemental material (ESM) Methods for further details. To further validate the results, fixed sections were blocked and another GLP-1 receptor antibody (MAb28141; R\&D Systems, Minneapolis, MN, USA) was used to confirm staining.

Study 2 Nine men and nine women with type 2 diabetes (defined as fasting plasma glucose $>6.94 \mathrm{mmol} / \mathrm{l}$ and/or $\mathrm{HbA}_{1 \mathrm{c}}>6.5 \%$ or $47.5 \mathrm{mmol} / \mathrm{mol}$ ) provided written informed consent to participate in this randomised, crossover, placebocontrolled, double-blind study, approved by the Beth Israel Deaconess Medical Center (BIDMC) Institutional Review Board (details in ESM Fig. 1). Participants first came in for a screening visit to the BIDMC clinical research centre (CRC) 
to ensure that they met the inclusion/exclusion criteria for the study. See ESM Methods for further details.

After the screening visit, participants were randomised to receive either liraglutide or placebo for the first arm of the crossover study. They received the opposite treatment in the second arm of the study. Thus, all 18 participants completed both the liraglutide and placebo visits. Both arms were identical and separated by a wash-out period (minimum 3 weeks, maximum 3 months). Each arm consisted of four visits in total. At the first three visits, separated from each other by 1 week, physical examination and blood draws were performed for monitoring safety. To minimise side effects (e.g. nausea), the dose of liraglutide was gradually increased $(0.6 \mathrm{mg}$ at the first visit, $1.2 \mathrm{mg}$ at the second and $1.8 \mathrm{mg}$ at the third). The final visit occurred 3 days after the third visit (i.e. when each participant had been on $1.8 \mathrm{mg}$ liraglutide for 3 days). At the final visit of each arm, participants attended the BIDMC CRC for a visit during which an overnight fast of at least $12 \mathrm{~h}$ was followed by an fMRI scan during the fasting state. Before and after the scan, participants used a visual analogue scale (VAS) to record subjective feelings of hunger, appetite and nausea. See ESM Methods for further details. Detailed anthropometric data (e.g. dual energy x-ray absorptiometry) were collected and resting metabolic rate was recorded.

Biochemical measurements and analysis Fasting blood was drawn by venipuncture by a registered nurse. Samples were immediately processed for plasma and serum isolation according to standard operating procedures and stored at $-80^{\circ} \mathrm{C}$ until analysis as previously described (ESM Table 1; [13, 14]). All samples and standards were assayed in duplicate and only results with a $\mathrm{CV}<15 \%$ were used.

Data were analysed using the Statistical Package for Social Sciences (SPSS; IBM Corporation; Armonk, NY, USA) v.19 and first summarised with descriptive statistics. The treatment effect was assessed using a general linear mixed model. The variables of treatment, visit and sequence were included in the model as fixed effects and patient-within-sequence was included as a random effect. Baseline values from the first visits in each arm were included as covariates. The values of anthropometric, clinical and laboratory variables at visit 4 and visit 8 were compared and used as dependent variables. Dependent variables that did not fulfil the normality assumptions were log-transformed before analysis. VAS and neurocognitive test results, collected at the endpoints for each phase (visits 4 and 8 ), were analysed with paired $t$ tests between the treatment and placebo conditions. See ESM Methods for further details.

fMRI protocol and analysis MRI scanning took place at the Center for Biomedical Imaging, Boston University School of Medicine, using a 3-T Philips Intera whole-body MRI (Philips Medical Systems, Best, the Netherlands) equipped with a 32- channel Synergy-L Sensitivity Encoding (SENSE) head coil. See ESM Methods for further details.

The fMRI protocol consisted of six runs during which participants viewed blocks of images of highly desirable foods high in energy or fat content (e.g. cakes, onion rings and other similar foods), less desirable foods low in energy or fat content (e.g. vegetables and fruits) or non-food items (e.g. flowers, rocks and trees). Participants reported their response to each image (how much they liked each image, on a scale of 1-3), using a response box held in their right hand. Blocks consisted of five images, each image being shown for $3 \mathrm{~s} \mathrm{(15s} \mathrm{total} \mathrm{for}$ each block), and 18 blocks were shown during each of the six runs.

Blood oxygenation level dependent (BOLD) data was preprocessed using SPM8 (Statistical Parametric Mapping; The Wellcome Trust Centre of Neuroimaging, London, UK). The contrast images (highly desirable $>$ less desirable food images and all food [both highly desirable and less desirable] cues $>$ non-food images) of the first-level analysis were used for the second-level group statistics. At the second level, we performed paired $t$ tests between participants' scans while on liraglutide and placebo for the two contrasts (highly desirable food cues $>$ less desirable food cues and all food cues $>$ nonfood cues). See ESM Methods for further details.

Neurocognitive testing Neurocognitive testing was done after the completion of the fasting MRI scan and the consumption of a meal on the CANTAB device (Cambridge Cognition, Cambridge, UK) (ESM Table 2).

\section{Results}

Study 1: expression of GLP-1 receptors in the human brain In the parietal cortex, GLP-1 receptors stained with various intensity all neurons (small pyramidal, large pyramidal and polymorphous cells) in all cases examined (Table 1, Figs 1 and 2). Large pyramidal cells were usually the most immunoreactive while the polymorphous cells stained weakly and in two cases faintly. Glial cells (oligodendrocytes and astrocytes) were not immunoreactive in any case.

In the hypothalamus, the arcuate nucleus, paraventricular nucleus and the complex of ventromedial nucleus neurons were immunopositive in the vast majority of the cases $(21 / 21,17 / 18$ and $18 / 18$, respectively). Stain intensity varied from weak to very strong, with the paraventricular and arcuate nuclei displaying strong immunoreactivity in eight cases each. Even though the neurons of the ventromedial nuclei were less immunoreactive, they were the structures that presented a distinct paranuclear (Golgi-like) stain pattern.

Area postrema and the dorsal motor nucleus of the vagus in the medulla oblongata expressed GLP-1 in all cases (10/10 and $11 / 11$, respectively) with the former displaying vigorous 
Table 1 Results of immunohistochemical analyses for areas of the hypothalamus, medulla oblongata and parietal cortex

\begin{tabular}{|c|c|c|c|c|c|c|c|c|c|c|c|c|}
\hline Case no. & Sex & SPC & LPC & PmC & NSol & $\mathrm{AP}$ & DMNV & SON & $\mathrm{SCN}$ & ARC & PVN & VMH \\
\hline 1 & M & ++ & + & + & & & & & & + & ++ & \\
\hline 2 & M & + & + & $-1+$ & - & & + & & & ++ & ++ & + \\
\hline 3 & $\mathrm{~F}$ & + & ++ & + & $-1+$ & & + & & & + & + & $-/+$ \\
\hline 4 & M & & & & & & & & & + & - & $-/+$ \\
\hline 5 & $\mathrm{~F}$ & ++ & ++ & + & & & & & ++ & ++ & + & ++ \\
\hline 6 & M & & & & & & & ++ & & ++ & ++ & $-/+$ \\
\hline 7 & M & & & & - & $-/+$ & $-1+$ & & & $-1+$ & ++ & $-/+$ \\
\hline 8 & M & $-1+$ & + & $-1+$ & & & & + & & + & $\begin{array}{l}+ \\
+\end{array}$ & ++ \\
\hline 9 & M & ++ & +++ & ++ & & & & & & ++ & ++ & + \\
\hline 10 & $\mathrm{~F}$ & ++ & +++ & ++ & & & & & & $\begin{array}{l}++ \\
++\end{array}$ & + & ++ \\
\hline 11 & M & & & & & + & $-1+$ & + & $-1+$ & + & - & $-/+$ \\
\hline 12 & $\mathrm{~F}$ & + & ++ & + & & & & & & ++ & ++ & + \\
\hline 13 & M & + & $-/+$ & $-/+$ & + & ++ & + & & & $-1+$ & + & $-/+$ \\
\hline 14 & $\mathrm{~F}$ & + & $-/+$ & $-/+$ & $-/+$ & + & ++ & & & & & \\
\hline 15 & M & & & & $-/+$ & ++ & + & ++ & & + & + & + \\
\hline 16 & $\mathrm{~F}$ & & & & $-/+$ & ++ & + & & & $\begin{array}{l}-1+ \\
+\end{array}$ & + & + \\
\hline 17 & M & & & & $-/+$ & ++ & & & & $\begin{array}{l}-1+ \\
+\end{array}$ & + & + \\
\hline 18 & M & & & & + & ++ & + & & & + & & + \\
\hline 19 & $\mathrm{~F}$ & $-1+$ & + & $-1+$ & & & & + & & $\begin{array}{l}+ \\
+\end{array}$ & $-1+$ & $\begin{array}{l}++ \\
+\end{array}$ \\
\hline 20 & $\mathrm{~F}$ & + & ++ & + & + & + & ++ & & & $\begin{array}{l}++ \\
++\end{array}$ & $\begin{array}{l}++ \\
+\end{array}$ & $\begin{array}{l}+ \\
+\end{array}$ \\
\hline 21 & $\mathrm{M}$ & + & ++ & + & - & + & + & + & & + & ++ & $-/+$ \\
\hline 22 & $\mathrm{~F}$ & & & & & & & + & & ++ & & \\
\hline
\end{tabular}

AP, area postrema; ARC, arcuate nucleus of the hypothalamus; DMNV, dorsal motor nucleus of the vagus; LPC, large pyramidal cells of the parietal cortex; NSol, nucleus solitarius; PmC, polymorphous cells of the parietal cortex; PVN, paraventricular nucleus of the hypothalamus; SPC, small pyramidal cells of the parietal cortex; $\mathrm{VMH}$, ventromedial nucleus of the hypothalamus; - , no staining; $-/+$, weak staining; +, moderate staining; ++, strong staining; +1, very strong staining; cells that are blank were not examined in that case; cells with two values show results of immunohistostaining in two consecutive paraffin blocks; e.g. ' ++++ ' indicates that tissues examined from two different paraffin blocks yielded strong staining in this area

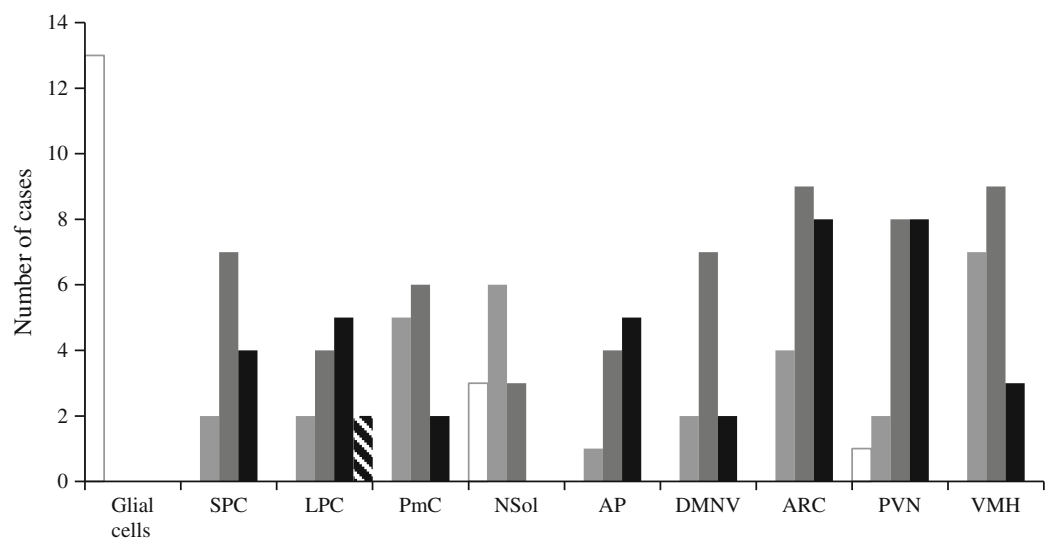

Fig. 1 Expression of GLP-1 receptors in human brain tissue. AP, area postrema; ARC, arcuate nucleus of the hypothalamus; DMNV, dorsal motor nucleus of the vagus; LPC, large pyramidal cells of the parietal cortex; NSol, nucleus solitarius; PmC, polymorphous cells of the parietal cortex; PVN, paraventricular nucleus of the hypothalamus; SPC, small pyramidal cells of the parietal cortex; VMH, ventromedial nucleus of the hypothalamus. White bars with grey border, no staining; light grey bars, weak staining; dark grey bars, mild staining; black bars, strong staining; diagonal stripe bars, very strong staining 


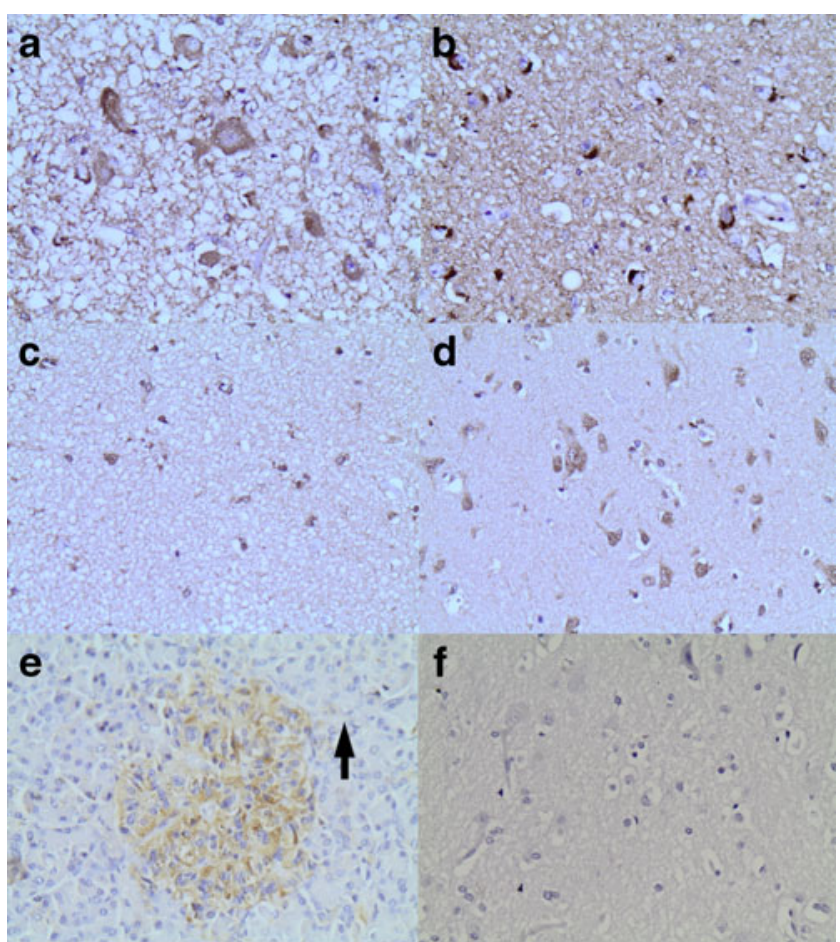

Fig. 2 Staining for the GLP-1 receptor (magnification $\times 400$ ) in the paraventricular nucleus of the hypothalamus (a), ventromedial hypothalamus (b), parietal cortex in small (c) and large pyramidal cells (d); staining in islets of Langerhans as a positive control (e) and glial and pyramidal cells without MAb 3F52 as a negative control (f). The arrow in (e) points to the non-neuroendocrine cells outside islets of Langerhans, which do not display immunopositivity, thus making them a valid positive control

stain intensity. The dorsal motor nucleus of the vagus moderately expressed the GLP-1 receptor antigen in the majority of the cases. Weak, almost faint, immunoreactivity for this antigen was also demonstrated in the nucleus solitarius ( $7 / 10$ positive, of which five were weak/faint).

Study 2: metabolic impact of liraglutide Eighteen adults ( 9 women; age $48.9 \pm 2.4$ years) with type 2 diabetes participated in this study. Weight, BMI and waist circumference remained unchanged with liraglutide treatment (Table 2). Immunoreactive GLP-1 was increased in response to liraglutide $(F$ statistic [degrees of freedom] $)(F[32])=40.8$; $p<0.001)$. The energy intake of participants was less while on $1.8 \mathrm{mg}$ liraglutide than while on placebo (placebo [averaged]: $7,456 \pm 803 \mathrm{~kJ} /$ day $[1,782 \pm 192 \mathrm{kcal} /$ day]; $0.6 \mathrm{mg}$ : $7,209 \pm 937 \mathrm{~kJ} /$ day $[1,723 \pm 224 \mathrm{kcal} /$ day $] ; 1.2 \mathrm{mg}: 6,862$ $\pm 586 \mathrm{~kJ} /$ day $[1,640 \pm 140 \mathrm{kcal} /$ day]; $1.8 \mathrm{mg}: 6,000 \pm 795 \mathrm{~kJ} /$ day $[1,434 \pm 190 \mathrm{kcal} /$ day]; $t$ statistic [degrees of freedom] $t[7]=2.11 ; p<0.07$, two-tailed; $p<0.03$, one-tailed). Fasting blood glucose levels improved with liraglutide treatment $(F[19]=24.5 ; p<0.001)$, although neither $\mathrm{HbA}_{1 \mathrm{c}}$ nor fructosamine improved within this short time period. The decrease in glucose remained significant in the treatment group when adjusted for BMI $(F[19]=22.7 ; p<0.001)$.

Side effects of liraglutide Nausea was reported equally by participants while receiving liraglutide and placebo at earlier visits $\left(n=7\right.$ participants in each arm; $38.9 \% ; \chi^{2}=0$; $p>0.999)$. VAS ratings of nausea were no different at the time of the MRIs $(t[17]=0.9 ; p<0.27$; liraglutide: $5.45 \pm 0.61$; placebo: $6.51 \pm 0.73)$. Although amylase showed no changes, lipase increased with liraglutide $(F[15]=12.1 ; p<0.003)$ within the normal range (Table 2).

Neurocognitive impact of liraglutide There were no significant changes in any of the neurocognitive testing results when comparing liraglutide with placebo (Table 3). With a pairedsamples $t$ test, participants showed less inferior parietal cortex activation $\left(297 \mathrm{~mm}^{3}\right.$; XYZ: $\left.-48,-26,46 ; z=4.13\right)$ in response to highly desirable vs less desirable food images while on $1.8 \mathrm{mg}$ liraglutide vs placebo at $p<0.001$, uncorrected (Fig. 3). In a secondary analysis, using small volume corrections on data from highly desirable vs less desirable images in the fasting state, we saw similar decreases in activation to those obtained with exenatide reported by van Bloemendaal et al [15] for insula and putamen (Fig. 4). Notably, we did not observe any changes in the hypothalamus.

Impact of neuroimaging Using the VAS for rating hunger, participants rated themselves as being fuller when taking liraglutide while fasting (liraglutide: $3.97 \pm 0.77$; placebo: $2.12 \pm 0.67 ; t[17]=3.25, p<0.006)$. The participants trended towards feeling that it would be less pleasant to eat during the fasting state on liraglutide, although this rating did not reach significance (liraglutide: $5.45 \pm 0.61$; placebo: $6.52 \pm 0.06$; $t[17]=1.94, p<0.09$, one-tailed based on an a priori hypothesis for a decrease). However, while receiving liraglutide, participants' ratings of how pleasant it would be to eat correlated with the parietal activation shown in the whole-brain analysis (i.e. those who showed the least activation of the parietal cortex in response to highly desirable food images thought it would be least pleasant to eat) $(r=0.820 ; p<0.024)$. In whole-brain regression analyses of the VAS ratings and brain activity during the fasting-state scan while on liraglutide, when comparing response to images of highly desirable vs less desirable food we observed that participants' ratings for concepts related to hunger and appetite showed a positive correlation with activation in many brain areas, including the parietal cortex, precuneus, cuneus and other areas of the occipital cortex (Table 4; Fig. 5a-c). On the other hand, participants' ratings of nausea inversely correlated with brain activation in the cingulate cortex, cuneus, precuneus and some areas of prefrontal cortex (Table 4; Fig. 5d). 
Table 2 Body composition data and hormonal measurements for each phase before (visit 1) and after 17 days of liraglutide or placebo (visit 4)

\begin{tabular}{|c|c|c|c|c|c|}
\hline \multirow[t]{2}{*}{ Variable } & \multicolumn{2}{|l|}{ Placebo } & \multicolumn{2}{|l|}{ Liraglutide } & \multirow[t]{2}{*}{$p$ value } \\
\hline & Visit 1 & Visit 4 & Visit 1 & Visit 4 & \\
\hline \multicolumn{6}{|l|}{ Anthropometry } \\
\hline BMI $\left(\mathrm{kg} / \mathrm{m}^{2}\right)$ & $31.2 \pm 1.7$ & $30.5 \pm 1.4$ & $31.9 \pm 1.7$ & $31.5 \pm 1.7$ & 0.94 \\
\hline Body weight (kg) & $93.4 \pm 4.8$ & $91.1 \pm 1.0$ & $94.7 \pm 4.7$ & $91.0 \pm 1.0$ & 0.42 \\
\hline WC iliac $(\mathrm{cm})$ & $105.1 \pm 3.5$ & $104.1 \pm 3.4$ & $106.8 \pm 3.5$ & $105.3 \pm 3.4$ & 0.34 \\
\hline Fat body mass (kg) & $30.9 \pm 3.1$ & $29.2 \pm 1.1$ & $31.5 \pm 2.9$ & $31.2 \pm 2.8$ & 0.69 \\
\hline Fat ( $\%$ of body mass) & $32.3 \pm 1.9$ & $32.6 \pm 1.9$ & $32.5 \pm 1.9$ & $32.7 \pm 2.0$ & 0.14 \\
\hline $\begin{array}{l}\text { Bone mass density } \\
\left(\mathrm{g} / \mathrm{cm}^{2}\right)\end{array}$ & $1.26 \pm 0.03$ & $1.26 \pm 0.03$ & $1.26 \pm 0.03$ & $1.26 \pm 0.03$ & 0.58 \\
\hline Lean body mass (kg) & $62.9 \pm 2.8$ & $63.0 \pm 2.6$ & $63.5 \pm 2.6$ & $62.4 \pm 2.6$ & 0.06 \\
\hline $\begin{array}{l}\text { Total energy intake (kJ/ } \\
\text { day) }[\mathrm{kcal} / \mathrm{day}]\end{array}$ & ND & $\begin{array}{l}7,456 \pm 803 \\
\quad(1,782 \pm 192)\end{array}$ & ND & $\begin{array}{l}6,000 \pm 795 \\
\quad(1,434 \pm 190)\end{array}$ & 0.07 \\
\hline \multicolumn{6}{|l|}{ Metabolic profile } \\
\hline GLP-1 (pmol/l) & $49.8 \pm 8.6$ & $38.2 \pm 1.2$ & $89.3 \pm 12.1$ & $912.3 \pm 1.2$ & $<0.001$ \\
\hline Glucose (mmol/l) & $6.60 \pm 0.35$ & $6.79 \pm 0.35$ & $6.92 \pm 0.34$ & $5.29 \pm 0.23$ & $<0.001$ \\
\hline Fructosamine $(\mu \mathrm{mol} / \mathrm{l})$ & $296.2 \pm 10.2$ & $273.5 \pm 8.5$ & $300.3 \pm 9.3$ & $271.2 \pm 8.3$ & 0.35 \\
\hline $\mathrm{HbA}_{1 \mathrm{c}}(\%[\mathrm{mmol} / \mathrm{mol}])$ & ND & $6.7 \pm 0.2(50 \pm 2)$ & ND & $6.7 \pm 0.2(50 \pm 2)$ & 0.82 \\
\hline Insulin $(\mu \mathrm{U} / \mathrm{ml})$ & $16.2 \pm 2.8$ & $11.5 \pm 2.6$ & $15.6 \pm 2.3$ & $11.2 \pm 1.4$ & 0.47 \\
\hline \multicolumn{6}{|l|}{ Safety tests } \\
\hline GGT (U/l) & $36 \pm 10.7$ & $29.5 \pm 8.5$ & $34.6 \pm 9.8$ & $33.6 \pm 11.5$ & 0.71 \\
\hline ALP (U/1) & $73.7 \pm 5.3$ & $64.2 \pm 4.0$ & $69.7 \pm 4.7$ & $64.2 \pm 4.1$ & 0.48 \\
\hline AST (U/1) & $21.2 \pm 2.5$ & $17.7 \pm 1.6$ & $18.1 \pm 1.1$ & $18.1 \pm 1.4$ & 0.74 \\
\hline ALT (U/l) & $24.6 \pm 4.7$ & $17.5 \pm 1.9$ & $19.9 \pm 1.9$ & $18.3 \pm 2.3$ & 0.72 \\
\hline Haematocrit (\%) & $43.2 \pm 2.9$ & $38 \pm 0.8$ & $39.6 \pm 0.8$ & $38.8 \pm 0.8$ & 0.11 \\
\hline Lymph (\%) & $33.2 \pm 1.7$ & $37 \pm 2.1$ & $37.3 \pm 2.3$ & $36.6 \pm 1.8$ & 0.81 \\
\hline Amylase (U/l) & $74.7 \pm 6.8$ & $73.8 \pm 8.4$ & $72.2 \pm 7.1$ & $76.32 \pm 8.1$ & 0.59 \\
\hline Lipase (U/1) & $28.5 \pm 2.5$ & $27.5 \pm 2.0$ & $29.2 \pm 2.0$ & $38.5 \pm 4.0$ & 0.003 \\
\hline
\end{tabular}

Data are shown as means \pm SEM

The $p$ value shown in the right column is from a general linear mixed model analysis of visit 4 values of the liraglutide and placebo phases. The variables of treatment, visit and sequence were included in the model as fixed effects and patient-within-sequence was included as a random effect. Baseline values from the first visits in each arm were included as covariates

ALP, alkaline phosphatase; ALT, alanine transaminase; AST, aspartate aminotransferase; GGT, $\gamma$-glutamyl transferase; ND, no data available; WC waist circumference

\section{Discussion}

This study provides novel information on the expression of GLP-1 receptors in human brains as well as on how liraglutide $1.8 \mathrm{mg}$ may influence short-term appetite regulation among individuals with diabetes. This is the first randomised, placebo-controlled, crossover trial to examine potential neural mechanisms activated by subcutaneous liraglutide therapy. More specifically, we observed changes in activation of the parietal cortex, insula and putamen in response to highly palatable food cues with liraglutide as compared with placebo. Our results suggest that liraglutide may exert central effects by decreasing attention to highly palatable foods, given decreased parietal cortex activation; additional central mechanisms will need to be explored more fully by means of larger trials and/or using higher doses of liraglutide.

Expression of GLP-1 receptors in the human brain We observed expression of GLP-1 receptors in the hypothalamic nuclei and medulla oblongata of humans, confirming the findings of studies in rodents and primates [10-12]. This suggests that GLP-1 analogues may confer some of their effects on appetite through actions on the hypothalamus. The identification of GLP-1 receptors in the area postrema may also explain why nausea is a relatively common side effect of GLP-1 analogues [16], considering the area postrema has a large role in nausea and vomiting [17].

Interestingly, we observed for the first time GLP-1 receptors on neurons in the parietal cortex, suggesting our findings 
Table 3 Outcomes of neurocognitive testing while on liraglutide vs placebo

\begin{tabular}{lllll}
\hline Test & Measure & Placebo & Liraglutide & $p$ value \\
\hline IED & Total errors & $59.88 \pm 9.45$ & $48.41 \pm 7.86$ & 0.22 \\
& Stages completed & $7.53 \pm 0.42$ & $7.88 \pm 0.30$ & 0.41 \\
SSP & Span length & $4.94 \pm 0.28$ & $4.76 \pm 0.32$ & 0.60 \\
SST & Go signal errors & $10.59 \pm 5.48$ & $5.71 \pm 1.16$ & 0.33 \\
& Stop success \% & $0.53 \pm 0.03$ & $0.47 \pm 0.03$ & 0.15 \\
& GoRT & $561.68 \pm 39.02$ & $525.38 \pm 39.57$ & 0.28 \\
& SSRT & $261.70 \pm 31.18$ & $234.85 \pm 28.23$ & 0.15 \\
SWM & Between errors & $62.53 \pm 2.83$ & $67.53 \pm 4.44$ & 0.29 \\
& Strategy & $33.06 \pm 0.74$ & $33.41 \pm 0.54$ & 0.69 \\
VRM & Free recall & $5.71 \pm 0.32$ & $6.18 \pm 0.53$ & 0.36 \\
& Recognition & $22.24 \pm 0.60$ & $22.18 \pm 0.49$ & 0.87 \\
& Delayed recognition & $21.59 \pm 0.73$ & $21.53 \pm 0.62$ & 0.93 \\
\hline
\end{tabular}

Data are shown as means \pm SEM

The $p$ values are from a paired-samples $t$ test between liraglutide and placebo

GoRT, median reaction time for go trials; IED, intra and extra dimensional set shift; SSP, spatial span; SSRT, stop signal reaction time; SST, stop signal task; SWM, spatial working memory; VRM, verbal recognition memory

of altered parietal activation with liraglutide could be a direct rather than an indirect effect (e.g. acting through hypothalamic circuitry) of the medication. This needs further study. Our findings are strengthened by the translational finding that activation of this area in fMRI was directly correlated with the desirability of food to patients. Our findings are also distinct from those of other studies, using the same antibody, reporting that there were no GLP-1 receptors in the parietal cortex of non-human primates [10]. Although we did not see changes in the hypothalamus in response to food cues while participants were taking liraglutide, this may be due to the limitations of MRI, due partly to the size of this structure and partly to its proximity to the sinuses, which
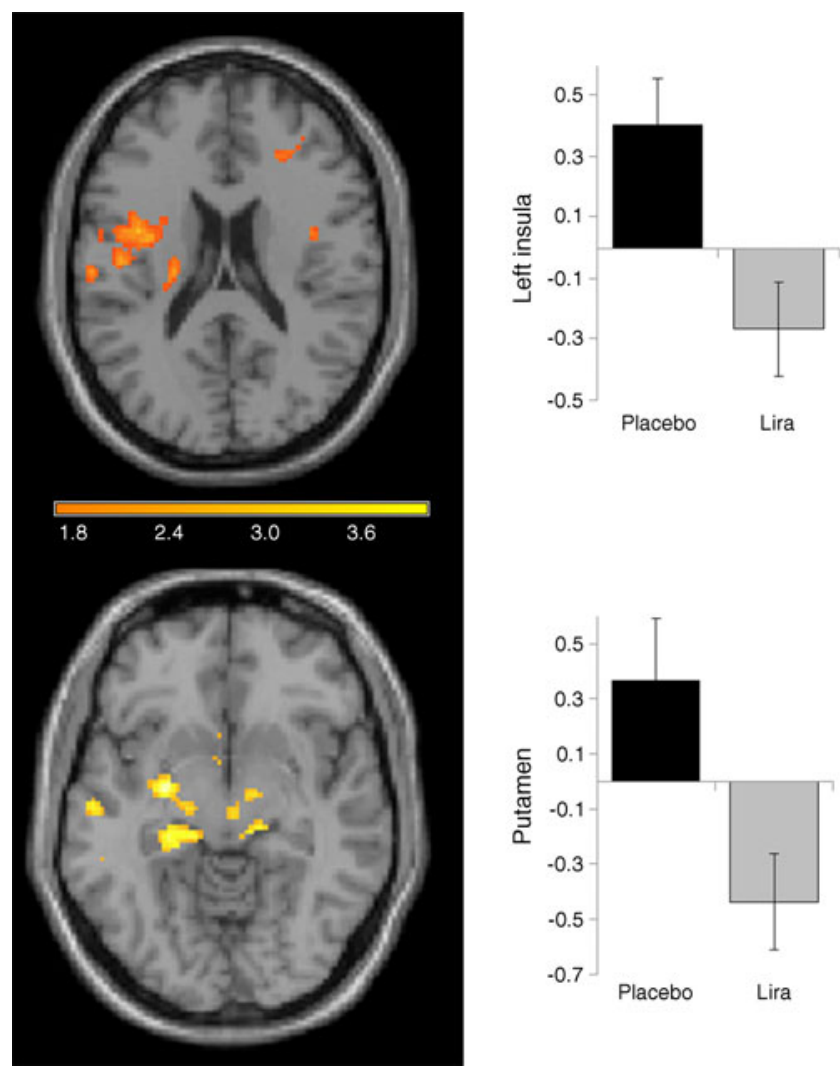

Fig. 4 In a secondary analysis, small volume corrections were performed on contrast images for highly vs less desirable food cues in the insula and putamen; to be compared with results from van Bloemendaal et al (Fig. 3) [15]. The $y$-axis represents effect size of the activation ( $z$ scores). BOLD contrasts are superimposed on a T1 structural image in neurological orientation. The colour bar represents voxel T value. Lira, liraglutide

creates artefacts [18]. As far as identification of GLP-1 in the human brain, Drucker and Asa described in 1988 GLP1 (also known as $G C G$ ) mRNA-positive neurons in the region of dorsal motor nucleus of the vagus nerve in the medulla oblongata [19]. Recently, GLP-1-immunopositive neurons within the human medulla oblongata have been observed in two patients [20]. Immunoreactivity was
Fig. 3 Brain activation in inferior parietal cortex in response to highly desirable compared with less desirable food images decreases with liraglutide treatment (whole-brain pairedsamples $t$ test). BOLD contrasts are superimposed on a $\mathrm{T} 1$ structural image in neurological orientation. The colour bar represents voxel $\mathrm{T}$ value

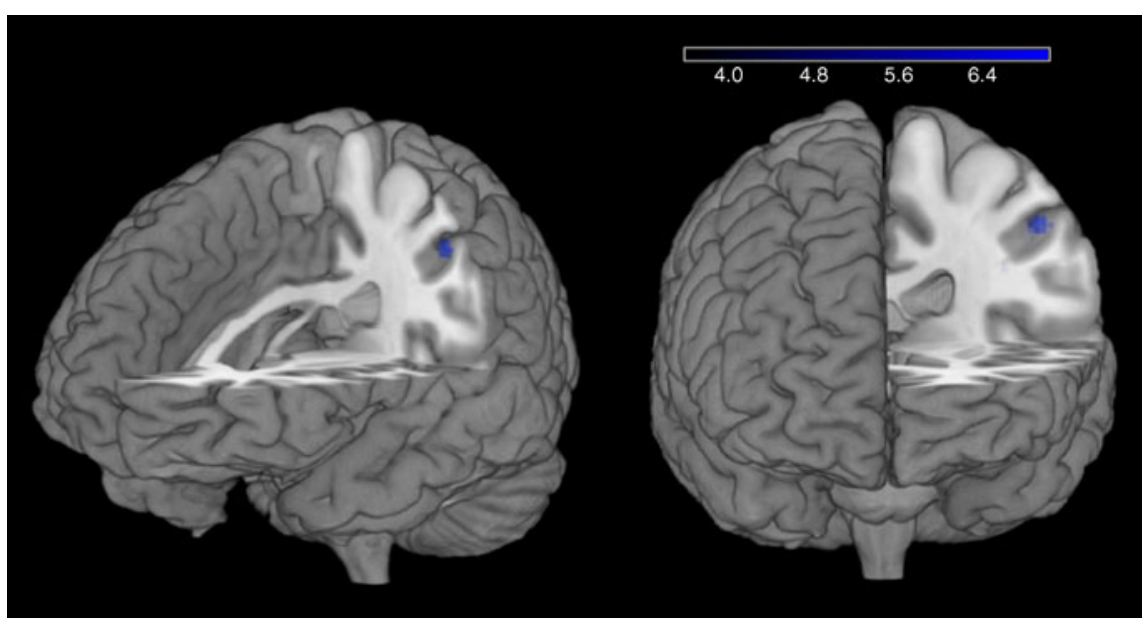


Table 4 Results from a wholebrain regression analysis and VAS data during the liraglutide fMRI session for the contrast of highly desirable vs less desirable food images in the fasting state

\begin{tabular}{llllll}
\hline $\begin{array}{l}\text { Cluster size } \\
\text { (voxels) }\end{array}$ & Voxel $z$ value & $\begin{array}{l}\text { MNI coordinates } \\
(\mathrm{mm})\end{array}$ & Side & Identified region & General brain area \\
\cline { 2 - 4 } & $x y \quad z$ & & \\
\hline
\end{tabular}

Correlation with 'How hungry do you feel right now?'

$\begin{array}{llllllll}30 & 4.25 & 8 & -54 & 56 & \mathrm{C} & \text { Precuneus } & \text { Precuneus } \\ 49 & 4.20 & -40 & -42 & 54 & \mathrm{~L} & \text { Inferior parietal G } & \text { Parietal cortex } \\ 70 & 4.05 & 24 & -58 & 56 & \mathrm{R} & \text { Precuneus } & \text { Precuneus }\end{array}$

Correlation with 'How pleasant would it be to eat right now?'

$\begin{array}{llllllll}214 * & 5.03^{\#} & 14 & -76 & -8 & \mathrm{R} & \text { Occipital G } & \text { Occipital cortex } \\ & 3.98 & 12 & -80 & 0 & \mathrm{R} & \text { Cuneus } & \text { Cuneus } \\ 100 & 4.09 & -28 & -92 & 8 & \mathrm{~L} & \text { Middle occipital G } & \text { Occipital cortex } \\ & 3.73 & -18 & -98 & 16 & \mathrm{~L} & \text { Cuneus } & \text { Cuneus } \\ 46 & 3.86 & 24 & -84 & 26 & \mathrm{R} & \text { Precuneus } & \text { Precuneus } \\ 71 & 3.83 & 6 & -86 & 18 & \mathrm{C} & \text { Cuneus } & \text { Cuneus } \\ 30 & 3.66 & -14 & -56 & -2 & \mathrm{~L} & \text { Occipital G } & \text { Occipital cortex } \\ & 3.33 & -14 & -64 & 2 & \mathrm{~L} & \text { Cuneus } & \text { Cuneus } \\ 30 & 3.62 & 32 & -88 & 2 & \mathrm{R} & \text { Middle occipital G } & \text { Occipital cortex } \\ 49 & 3.56 & -58 & -32 & -10 & \mathrm{~L} & \text { Middle temporal G } & \text { Temporal lobe } \\ 35 & 3.48 & -36 & -84 & 0 & \mathrm{~L} & \text { Middle occipital G } & \text { Occipital cortex }\end{array}$

Correlation with 'How much do you think you could eat right now?'

$\begin{array}{llllllll}78 & 4.24 & 24 & -58 & 56 & \mathrm{R} & \begin{array}{l}\text { Superior parietal } \\ \text { lobule }\end{array} & \text { Parietal cortex } \\ 115 & 3.96 & -18 & -92 & 24 & \mathrm{~L} & \text { Cuneus } & \text { Cuneus } \\ & 3.77 & -26 & -86 & 14 & \mathrm{~L} & \text { Middle occipital G } & \text { Occipital cortex } \\ 55 & 3.64 & 6 & -92 & 20 & \mathrm{C} & \text { Cuneus } & \text { Cuneus }\end{array}$

Inverse correlation with 'How nauseous do you feel right now?'

\begin{tabular}{llllllll}
$465^{*}$ & 4.7 & 20 & -16 & 52 & $\mathrm{R}$ & Cingulate G & Cingulate cortex \\
105 & 4.53 & -12 & -50 & 28 & $\mathrm{~L}$ & Cingulate G & Cingulate cortex \\
& 4.25 & -6 & -56 & 18 & $\mathrm{C}$ & $\begin{array}{l}\text { Posterior cingulate G } \\
\text { Cingulate cortex }\end{array}$ & $\begin{array}{c}\text { Somatosensory } \\
\text { cortex }\end{array}$ \\
62 & 4.47 & 38 & -12 & 28 & $\mathrm{R}$ & Postcentral G & Temporal lobe \\
98 & 4.05 & -50 & -38 & 4 & $\mathrm{~L}$ & Superior temporal G & Hippocampus \\
& 3.84 & -36 & -30 & -2 & $\mathrm{~L}$ & Hippocampus & Cingulate cortex \\
$162^{*}$ & 3.86 & 18 & -48 & 26 & $\mathrm{R}$ & Cingulate G & Temporal lobe \\
31 & 3.83 & 52 & -50 & 18 & $\mathrm{R}$ & Superior temporal G \\
35 & 3.82 & 12 & -84 & 20 & $\mathrm{R}$ & Cuneus & Cuneus \\
49 & 3.74 & -6 & -72 & 18 & $\mathrm{C}$ & Precuneus & Precuneus \\
32 & 3.68 & 30 & -58 & 22 & $\mathrm{R}$ & Cuneus & Cuneus \\
78 & 3.68 & -24 & -10 & 44 & $\mathrm{~L}$ & Superior frontal G & Superior frontal \\
& 3.29 & -30 & -8 & 52 & $\mathrm{~L}$ & Precentral G & Sensorimotor \\
\hline
\end{tabular}

Clusters which pass a threshold of $p<0.001$, uncorrected and have an extent of at least 20 voxels are shown. Peaks shown for clusters are the most significant along the same identified region

Statistical threshold: $p<0.001$, uncorrected; extent, 30 voxels; ${ }^{*} p<0.05$, family-wise error corrected for cluster; ${ }^{\dagger} p<0.05$, family-wise error corrected for peak

C, centre, G, gyrus; L, left; R, right

displayed in the nucleus of the solitary tract, in area postrema and in the dorsal motor nucleus of the vagus nerve. We will expand our findings to determine whether the GLP-1 receptor also exists in these areas and explore its presence in more patients.
Metabolic impact of liraglutide In our trial GLP-1 immunoreactivity in the circulation of individuals increased in a stepwise manner with increasing doses of liraglutide. Additionally, fasting blood sugar levels improved without weight loss in this short course of $1.8 \mathrm{mg}$ liraglutide therapy. 


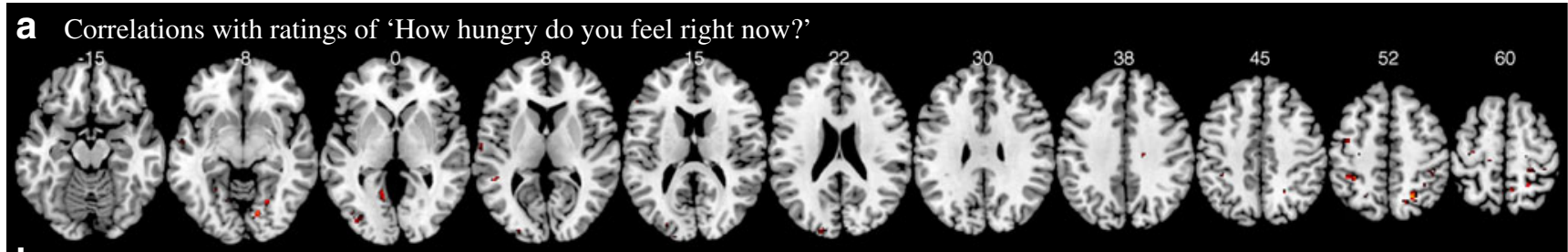

b Correlations with ratings of 'How pleasant would it be to eat right now?'

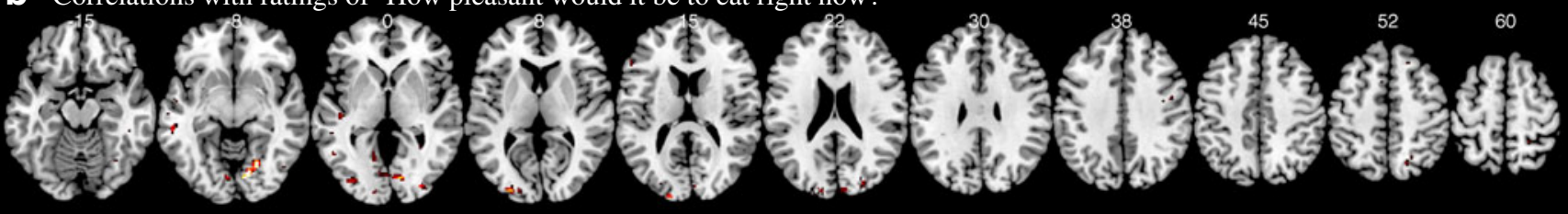

C Correlations with ratings of 'How much do you think you could eat right now?'

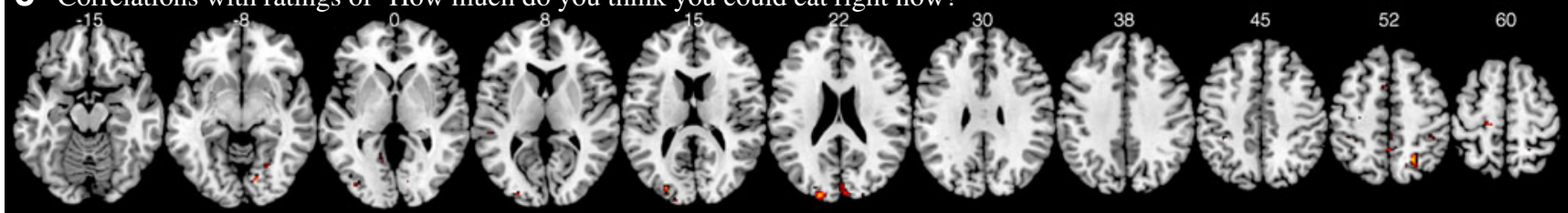

d Negative correlations with ratings of 'How nauseous do you feel right now?'

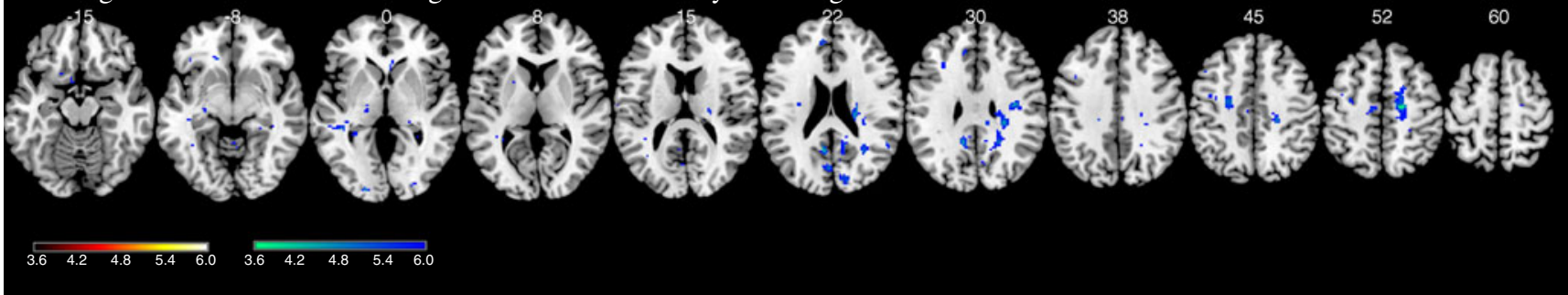

Fig. 5 Results from a whole-brain regression with VAS data during the liraglutide fMRI session for the contrast of highly desirable vs less desirable food images in the fasting state. Participants' ratings of the VAS questions along a $10 \mathrm{~cm}$ line were regressed with whole-brain activations: 'How hungry do you feel right now?' (a); 'How pleasant would it be to eat right now?' (b); 'How much do you think you could eat right now?' (c) and 'How nauseous do you feel right now?' (d). Hot colours show positive correlations, cold colours show negative correlations. BOLD contrasts are superimposed on a $\mathrm{T} 1$ structural image in axial sections from $z=-15$ to $z=60$, in neurological orientation. The colour bars represent voxel $\mathrm{T}$ value

weight loss in another fMRI study, suggesting that decreased attention to these images leads to greater weight loss [24]. Additionally, our participants rated themselves as feeling fuller in the fasting state while receiving liraglutide and had a lower energy intake with increasing liraglutide doses, altogether suggesting that they would indeed lose weight. Those with the least inferior parietal activation identified in the whole-brain analysis also reported it would be less pleasant to eat while on liraglutide during the fasting state, further supporting this notion. This change in activation may indicate neural changes that could proceed to and predict future weight loss with liraglutide, a notion to be fully evaluated by longterm studies.

Further supporting these findings are our whole-brain regression results, which show that activation of areas related to attention and saliency processing, including the occipital cortex, cuneus, precuneus and parietal cortex, were correlated with ratings of hunger and appetite. Greater activation in these areas in response to highly desirable vs less desirable food cues during the fasting state while on liraglutide were 
associated with higher levels of hunger, larger appetite and a feeling of how pleasant it would be to eat. These occipital areas (cuneus, precuneus and occipital cortex) are known to respond to food cues and this has been attributed to the emotional salience of food cues $[25,26]$. Indeed, increased activation of occipital cortex has also been linked with emotional images $[27,28]$. This may indicate individual differences in response to liraglutide, where participants who have decreased activation in these areas show less hunger and appetite. Interestingly, ratings of nausea while on liraglutide correlated inversely with activations in some of the same attentionrelated brain areas, likely indicating that participants are less interested in food items while feeling nauseous, as would be expected. We also report changes in activation in sensorimotor areas, including the cingulate, superior frontal and somatosensory cortex, which are often linked with feelings of nausea $[29,30]$. These may be deactivated in response to highly desirable food cues simply due to the feeling of nausea and lack of desirability of these types of foods when one is feeling nauseous. Additionally, nausea is a side effect of medication, so these changes might be a result of increased medication effects for certain individuals at 17 days.

There are limited other studies examining the effects of other GLP-1 analogues in the human brain. One study has demonstrated changes in resting-state hypothalamic connectivity with a single continuous infusion of exenatide in obese responders (e.g. those who consumed less food at a subsequent ad libitum buffet) [31]. A recent study using exenatide found changes in brain areas of the reward system, including the insula and orbital frontal cortex, with a similar task [15]. Nausea could be considered a confounder in that study which used a single dose, whereas in our study, nausea had subsided for most patients but weight loss had not yet occurred [15]. We did observe similar activation in the insula and putamen with the same small volume correction on our data. It remains to be seen whether statistically significant results would also be observed with a higher dose of liraglutide (3 $\mathrm{mg}$ ) or more participants. More recently, another study comparing liraglutide with insulin showed similar decreases in activation to food cues after 10 days, but not 12 weeks, in the insula and putamen [32]. It is well known that the insula is involved in saliency processing and satiety [33-35]. Insular activity has been shown to be modulated with ratings of hunger and fasted/ fed states [34, 35], indicating a role in the salience of food images similar to the parietal cortex, consistent with its activity during non-food salient stimuli [36]. Some studies have suggested that the putamen may be involved in the reward processing of food [15, 37-39] and have shown links between activation in the putamen and future weight gain [38]. Thus, since these areas decrease in activation on liraglutide, we would assume that participants find highly palatable foods less rewarding or relevant while on liraglutide. We report such data as only preliminary, however, and they need to be confirmed by future studies.

Limitations and future directions We demonstrate a central role for GLP-1 analogues in mediating weight loss in humans. Results from immunohistochemical studies support the findings of our fMRI study, suggesting that liraglutide likely acts through the parietal cortex directly to decrease attention to highly palatable food cues. Due to the crossover design of our study with randomly assigned sequence, we are confident that potential covariates are well-controlled and data are appropriately analysed, since each individual serves as his or her own control. However, the strength of our findings could be amplified by the addition of more participants and/or with studies of longer duration.

We did not see any changes in fMRI activation of the hypothalamus with liraglutide, in contrast to immunohistochemistry findings in rodents and primates $[10,12]$. This may be due to differences in terms of control of eating between rodents and humans; in the latter the homeostatic control of eating is often overridden by more complex cortical systems regulating the rewarding value of food [18]. This may also be a limitation of fMRI in general, as the hypothalamus is difficult to detect with typical fMRI measures due in part to its small size and in part to its proximity to the sinuses, which create artefacts [18].

The fMRI outcomes of our study need to be studied in different time frames after administration to explore possible sequential effects (e.g. changes at first dose and after longerterm use). Additionally, comparative studies to explore potential differences between types of GLP-1 analogues would be useful. Also, different doses (e.g. $3.0 \mathrm{mg}$ vs $1.8 \mathrm{mg}$ liraglutide) need to be examined further. It is possible that more pronounced changes could have been observed with the newly recommended higher dose of liraglutide $(3.0 \mathrm{mg})$, which has been shown to cause weight loss more dramatically [40]. In fact, despite no difference in gastric emptying or nausea, the $3.0 \mathrm{mg}$ dose of liraglutide caused greater weight loss than the $1.8 \mathrm{mg}$ dose, suggesting greater central/brain changes [40]. Thus, future studies need to examine how this higher dose alters brain activations.

We did not see any significant changes with respect to neurocognition; the variable closest to significance was the stop signal reaction time (i.e. the most appropriate test as per our a priori hypotheses), which can be used for future power calculations with higher doses and/or longer duration. The stop signal reaction time is a measure of inhibitory control, where a lower time indicates greater inhibitory control, which has been shown to be impaired with obesity [41]. This may indicate that with higher doses and/or more participants, liraglutide may increase inhibitory control, but this will need to be confirmed. 
Acknowledgements We acknowledge L. B. Knudsen (Novo Nordisk A/S, Maaloev, Denmark) for providing the GLP-1 receptor antibody (MAb 3F52).

Funding The project was supported by Harvard Clinical and Translational Science Center Grant UL1 RR025758 from the National Center for Research Resources. Novo Nordisk supported the study through an Investigator-Initiated Study grant and supplied liraglutide/placebo. They approved the design of the study, but had no role in study design, conduct of the study, collection, management, analysis and interpretation of the data or the preparation, review or approval of the manuscript. OMF is supported by a training grant through the NICHD 5T32HD052961.

Contribution statement OMF wrote the manuscript, analysed the data and researched data. MS and MAT wrote and edited the manuscript and researched data. B-JK and AG analysed the data and reviewed the manuscript. AS-E, FD, AF, JB, AS, NK, CL, ST-B and BT researched data and reviewed the manuscript. CSM composed the protocol, researched data and reviewed and edited the manuscript. OMF is the guarantor of this work. All authors approved the final version of the manuscript.

Duality of interest CSM has served on the scientific advisory board and is a shareholder of Novo Nordisk. All other authors declare that there is no duality of interest associated with their contribution to this manuscript.

\section{References}

1. Narayan KM, Boyle JP, Thompson TJ, Sorensen SW, Williamson DF (2003) Lifetime risk for diabetes mellitus in the United States. JAMA 290:1884-1890

2. Johnelle Sparks P, Bollinger M (2011) A demographic profile of obesity in the adult and veteran US populations in 2008. Popul Res Policy Rev 13:211-233

3. Aronis KN, Tsoukas MA, Mantzoros CS (2014) Potential cardioprotective action of GLP-1: from bench to bedside. Metabolism 63:979-988

4. Vilsboll T, Zdravkovic M, Le-Thi T et al (2007) Liraglutide, a longacting human glucagon-like peptide-1 analog, given as monotherapy significantly improves glycemic control and lowers body weight without risk of hypoglycemia in patients with type 2 diabetes. Diabetes Care 30:1608-1610

5. Parks M, Rosebraugh C (2010) Weighing risks and benefits of liraglutide - the FDA s review of a new antidiabetic therapy. $\mathrm{N}$ Engl J Med 362:774-777

6. Larsen PJ, Tang-Christensen M, Holst JJ, Orskov C (1997) Distribution of glucagon-like peptide-1 and other preproglucagonderived peptides in the rat hypothalamus and brainstem. Neuroscience 77:257-270

7. Hunter K, Holscher C (2012) Drugs developed to treat diabetes, liraglutide and lixisenatide, cross the blood brain barrier and enhance neurogenesis. BMC Neurosci 13:33

8. Holst JJ (2007) The physiology of glucagon-like peptide 1. Physiol Rev 87:1409-1439

9. Sisley S, Gutierrez-Aguilar R, Scott M, D Alessio DA, Sandoval DA, Seeley RJ (2014) Neuronal GLP1R mediates liraglutide s anorectic but not glucose-lowering effect. J Clin Invest 124:24562463

10. Heppner KM, Kirigiti M, Secher A et al (2015) Expression and distribution of glucagon-like peptide-1 receptor mRNA, protein and binding in the male nonhuman primate (Macaca mulatta) brain Endocrinology 156:255-267

11. Wang XF, Liu JJ, Xia J, Liu J, Mirabella V, Pang ZP (2015) Endogenous glucagon-like peptide-1 suppresses high-fat food intake by reducing synaptic drive onto mesolimbic dopamine neurons. Cell Rep 12:726-733

12. Pyke C, Heller RS, Kirk RK et al (2014) GLP-1 receptor localization in monkey and human tissue: novel distribution revealed with extensively validated monoclonal antibody. Endocrinology 155 : $1280-1290$

13. Farr OM, Ko BJ, Joung KE et al (2015) Posttraumatic stress disorder, alone or additively with early life adversity, is associated with obesity and cardiometabolic risk. Nutr Metab Cardiovasc Dis 25: 479-488

14. Welt CK, Chan JL, Bullen J et al (2004) Recombinant human leptin in women with hypothalamic amenorrhea. N Engl J Med 351:987997

15. van Bloemendaal L, RG IJ, Ten Kulve JS et al (2014) GLP-1 receptor activation modulates appetite- and reward-related brain areas in humans. Diabetes 63: 4186-4196

16. Filippatos TD, Panagiotopoulou TV, Elisaf MS (2014) Adverse effects of GLP-1 receptor agonists. Rev Diabet Stud 11:202-230

17. Horn CC (2014) The medical implications of gastrointestinal vagal afferent pathways in nausea and vomiting. Curr Pharm Des 20: 2703-2712

18. Salem V, Dhillo WS (2015) Imaging in endocrinology: the use of functional MRI to study the endocrinology of appetite. Eur J Endocrinol 173:R59-R68

19. Drucker DJ, Asa S (1988) Glucagon gene expression in vertebrate brain. J Biol Chem 263:13475-13478

20. Zheng H, Cai L, Rinaman L (2015) Distribution of glucagon-like peptide 1-immunopositive neurons in human caudal medulla. Brain Struct Funct 220:1213-1219

21. Corbetta M, Shulman GL (2002) Control of goal-directed and stimulus-driven attention in the brain. Nat Rev Neurosci 3:201-215

22. McFadden KL, Cornier MA, Melanson EL, Bechtell JL, Tregellas JR (2013) Effects of exercise on resting-state default mode and salience network activity in overweight/obese adults. Neuroreport 24:866-871

23. Capotosto P, Tosoni A, Spadone S et al (2013) Anatomical segregation of visual selection mechanisms in human parietal cortex. J Neurosci 33:6225-6229

24. Murdaugh DL, Cox JE, Cook EW 3rd, Weller RE (2012) fMRI reactivity to high-calorie food pictures predicts short- and longterm outcome in a weight-loss program. Neuroimage 59:2709 2721

25. Fuhrer D, Zysset S, Stumvoll M (2008) Brain activity in hunger and satiety: an exploratory visually stimulated FMRI study. Obes (Silver Spring, Md) 16:945-950

26. Schur EA, Kleinhans NM, Goldberg J, Buchwald D, Schwartz MW, Maravilla K (2005) Activation in brain energy regulation and reward centers by food cues varies with choice of visual stimulus. Int J Obes 33:653-661

27. Lang PJ, Bradley MM (2010) Emotion and the motivational brain. Biol Psychol 84:437-450

28. Lang PJ, Bradley MM, Fitzsimmons JR et al (1998) Emotional arousal and activation of the visual cortex: an fMRI analysis. Psychophysiology 35:199-210

29. Goldberg II, Harel M, Malach R (2006) When the brain loses its self: prefrontal inactivation during sensorimotor processing. Neuron 50:329-339

30. Napadow V, Sheehan JD, Kim J et al (2013) The brain circuitry underlying the temporal evolution of nausea in humans. Cereb Cortex 23:806-813 
31. Schlogl H, Kabisch S, Horstmann A et al (2013) Exenatide-induced reduction in energy intake is associated with increase in hypothalamic connectivity. Diabetes Care 36:1933-1940

32. Ten Kulve JS, Veltman DJ, van Bloemendaal L et al (2016) Liraglutide reduces CNS activation in response to visual food cues only after short-term treatment in patients with type 2 diabetes. Diabetes Care 39:214-221

33. De Silva A, Salem V, Long CJ et al (2011) The gut hormones PYY 3-36 and GLP-1 7-36 amide reduce food intake and modulate brain activity in appetite centers in humans. Cell Metab 14:700-706

34. Porubska K, Veit R, Preissl H, Fritsche A, Birbaumer N (2006) Subjective feeling of appetite modulates brain activity: an fMRI study. Neuroimage 32:1273-1280

35. St-Onge MP, Sy M, Heymsfield SB, Hirsch J (2005) Human cortical specialization for food: a functional magnetic resonance imaging investigation. J Nutr 135:1014-1018

36. Menon V, Uddin LQ (2010) Saliency, switching, attention and control: a network model of insula function. Brain Struct Funct 214: 655-667
37. Deckersbach T, Das SK, Urban LE et al (2014) Pilot randomized trial demonstrating reversal of obesity-related abnormalities in reward system responsivity to food cues with a behavioral intervention. Nutr Diabetes 4:e129

38. Burger KS, Stice E (2014) Greater striatopallidal adaptive coding during cue-reward learning and food reward habituation predict future weight gain. NeuroImage 99:122-128

39. Spetter MS, de Graaf C, Mars M, Viergever MA, Smeets PA (2014) The sum of its parts - effects of gastric distention, nutrient content and sensory stimulation on brain activation. PLoS One 9: e90872

40. Wadden TA, Hollander P, Klein S, et al. (2013) Weight maintenance and additional weight loss with liraglutide after low-calorie-dietinduced weight loss: the SCALE Maintenance randomized study. Int J Obes (Lond) 37: 1443-1451

41. Levitan RD, Rivera J, Silveira PP et al (2015) Gender differences in the association between stop-signal reaction times, body mass indices and/or spontaneous food intake in pre-school children: an early model of compromised inhibitory control and obesity. Int J Obes (Lond) 39:614-619 